\title{
The nucleus of Comet 9P/Tempel 1: Shape and geology from two flybys
}

\author{
P. Thomas ${ }^{\mathrm{a}, *}$, M. A'Hearn ${ }^{\mathrm{b}}$, M.J.S. Belton ${ }^{\mathrm{c}}$, D. Brownlee ${ }^{\mathrm{d}}$, B. Carcich ${ }^{\mathrm{a}}$, B. Hermalyn ${ }^{\mathrm{e}}$, K. Klaasen ${ }^{\mathrm{f}}$,

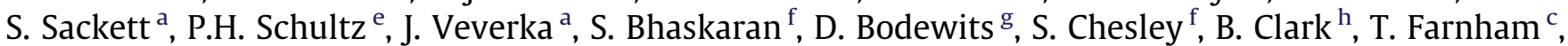 \\ O. Groussin ${ }^{\mathrm{i}}$, A. Harris ${ }^{\mathrm{j}}$, J. Kissel ${ }^{\mathrm{k}}$, J.-Y. Li ${ }^{\mathrm{b}}$, K. Meech ${ }^{1}$, J. Melosh ${ }^{\mathrm{m}}$, A. Quick ${ }^{\mathrm{n}}$, J. Richardson ${ }^{\mathrm{m}}$, \\ J. Sunshine ${ }^{\mathrm{b}}$, D. Wellnitz ${ }^{\mathrm{b}}$ \\ ${ }^{a}$ Cornell University, Ithaca, NY 14853, USA \\ ${ }^{\mathrm{b}}$ University of Maryland, College Park, MD 20742, USA \\ ${ }^{\mathrm{c}}$ Belton Space Exploration Initiatives, Tucson, AZ 85716, USA \\ ${ }^{\mathrm{d}}$ University of Washington, Seattle, WA 98195, USA \\ ${ }^{\mathrm{e}}$ Brown University, Providence, RI 02912, USA \\ ${ }^{\mathrm{f}}$ Jet Propulsion Laboratory, California Institute of Technology, Pasadena, CA 91109, USA \\ ${ }^{\mathrm{g}}$ NASA Goddard Space Flight Center, Greenbelt, MD 20771, USA \\ ${ }^{\mathrm{h}}$ Space Science Institute, Boulder, CO 80301, USA \\ ${ }^{\mathrm{i}}$ Laboratoire d'Astrophysique de Marseille, 13388 Marseille Cedex 13, France \\ ${ }^{\mathrm{j}}$ Space Science Institute, La Canada, CA 91011, USA \\ ${ }^{\mathrm{k}}$ Max Planck Institute for Solar System Research, Katlenburg-Lindau, Germany \\ ${ }^{1}$ University of Hawaii, Honolulu, HI 96822, USA \\ ${ }^{\mathrm{m}}$ Purdue University, Lafayette, IN 47907, USA \\ ${ }^{\mathrm{n}}$ University of Rochester, Rochester, NY 14627, USA
}

\section{A R T I C L E I N F O}

\section{Article history:}

Available online 7 March 2012

\section{Keywords:}

Comet Tempel 1

Comets, Nucleus

Geological processes

\begin{abstract}
A B S T R A C T
The nucleus of comet Tempel 1 has been investigated at close range during two spacecraft missions separated by one comet orbit of the Sun, $5 \frac{1}{2} 2$ years. The combined imaging covers $\sim 70 \%$ of the surface of this object which has a mean radius of $2.83 \pm 0.1 \mathrm{~km}$. The surface can be divided into two terrain types: rough, pitted terrain and smoother regions of varying local topography. The rough surface has round depressions from resolution limits ( $\sim 10 \mathrm{~m} /$ pixel) up to $\sim 1 \mathrm{~km}$ across, spanning forms from crisp steep-walled pits, to subtle albedo rings, to topographic rings, with all ranges of morphologic gradation. Three gravitationally low regions of the comet have smoother terrain, parts of which appear to be deposits from minimally modified flows, with other parts likely to be heavily eroded portions of multiple layer piles. Changes observed between the two missions are primarily due to backwasting of scarps bounding one of these probable flow deposits. This style of erosion is also suggested by remnant mesa forms in other areas of smoother terrain. The two distinct terrains suggest either an evolutionary change in processes, topographically-controlled processes, or a continuing interaction of erosion and deposition.
\end{abstract}

(c) 2012 Elsevier Inc. All rights reserved.

\section{Introduction}

The February 2011 flyby of comet Tempel 1 by the Stardust spacecraft as the Stardust-NExT mission (SDN) constituted the first revisit of a comet by a spacecraft (Veverka et al., 2012). The Deep Impact mission (DI) in 2005 provided image coverage of $~ 40 \%$ of the surface, determined many physical properties, and showed a surprisingly complex array of surface forms, including smooth regions thought to be the result of flows depositing materials on the surface (A'Hearn et al., 2005a,b; Belton and Melosh, 2009). Given the restricted image coverage, it was not clear what the truly

\footnotetext{
* Corresponding author.

E-mail address: pct2@cornell.edu (P. Thomas).
}

typical topography and dominant surface processes of the comet were, and how distinct Tempel 1 really was from the only other comet seen with anywhere nearly comparable resolution, Wild 2 (Brownlee et al., 2004). The DI images hinted at thick layering in the body of the comet along with more superficial, possibly flowrelated layers (Thomas et al., 2007; Belton et al., 2007), but how pervasive these were remained uncertain. The revisit to Tempel 1 allowed more of the nucleus to be mapped providing a much better assessment of the types of terrain as well as detection of changes during one solar orbit, presumably formed during one perihelion passage.

This paper presents the basic image maps, shape, short descriptions of the geological features, and a brief interpretation of the history of the comet's surface including estimates of rates of surface modification by erosion and deposition. 


\section{Data and methods}

Data are from the DI and SDN missions. The DI spacecraft and its instruments are described in A'Hearn et al. (2005a) and Hampton et al. (2005); the flyby is summarized in A'Hearn et al. (2005b). Most DI work reported here is done with the Medium Resolution Instrument (MRI) data, which had a pixel scale of $7 \mathrm{~m}$ at the closest approach of $\sim 700 \mathrm{~km}$, and the nearly identical Impactor Targeting Sensor (ITS), which obtained higher resolution, but smaller, windowed images just before impact. Most useful data were taken within $1500 \mathrm{~km}$ and spanned phase angles of $63-70^{\circ}$. The High Resolution Instrument (HRI), which has nominally five times better resolution than MRI, was determined to be out of focus after launch (Klaasen et al., 2008). Deconvolution (Lindler et al., 2012) can render some of these images suitable for detecting smaller forms than are visible in the MRI data, but this processing often introduces artifacts such that considerable care is required in interpreting these data.

The SDN data are from the NAVCAM instrument (Brownlee et al., 2004; Newburn et al., 2003; Klaasen et al., 2012). The flyby of Tempel 1 is discussed in Veverka et al. (2012). Closest approach images from $\sim 180 \mathrm{~km}$ have pixel scales of $11 \mathrm{~m}$. Phase angles of the best data cover $15-60^{\circ}$. Navigation data in the form of "SPICE" kernels (Semenov et al., 2004; Semenov and Acton, 2006) are the basis for all geometric work on the comet.
Determination of the shape and accurate relative positioning of the images relies upon stereo control points (Fig. 1) with the usual image pointing adjustments (Thomas et al., 2002). There are 480 manually measured points in the $\sim 70 \%$ of the comet that is well observed. Residuals (predicted image location vs. actual image location) have rms values of 0.42 pixels, or typically $\sim 6 \mathrm{~m}$.

Mapping of features and projection of images is enabled by image cubes that store latitude, longitude, radius, incidence, and emission angles at each pixel. Line and sample coordinates of features that have been individually marked or the original image data can then be arbitrarily projected.

Quantities such as gravity and slope are calculated using an assumed uniform density of $400 \mathrm{~kg} / \mathrm{m}^{-3}$ for the nucleus (Richardson et al., 2007) and calculated rotation vectors. The slowly varying spin period of $\sim 40$ h (Belton et al., 2011) imposes relatively small additional accelerations on the surface gravity field. These relatively small rotational accelerations mean that uncertainties in the mean density have little effect on calculations of relative potential energy across the surface. In this paper we use a single spin period of $40.7374 \mathrm{~h}$ for analysis of the data from both DI and SDN. This is an arbitrary value useful only in the synchronization of partial rotations. "Topography" is calculated as dynamic height, the surface potential energy divided by an average acceleration (Vanicek and Krakiwsky, 1986, p. 369; Thomas, 1993). "Slope" is the angle between surface normal and the local acceleration vectors.


Fig. 1. Shape of Tempel 1 nucleus. (A) Location of limbs and control points restricting the shape model of Tempel 1 . Simple cylindrical projection. Jagged nature of limb lines

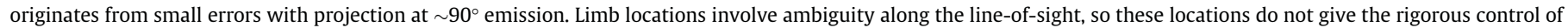
stereo points; they do limit the shape, however. (B) Model of the shape of Tempel 1 from different perspectives with gravitational heights projected on the surface. 


\section{Spin vector}

Tempel 1 has a changing spin period of approximately $40 \mathrm{~h}$ (period decreases approximately by $15 \mathrm{~min}$ per perihelion passage) and precession of the spin vector is expected to be small (Belton et al., 2011). The two spacecraft flybys provide two snapshots of the nucleus' orientation that may be used to calculate an effective rotation between the orientations of the nucleus in the 2005 and 2011 observations. Manual selection of 480 control points provides the basis for solving for both the effective pole orientation and for body-centered coordinates of a shape model. An average spin rate of $212.09019 \mathrm{deg} / \mathrm{d}$, which probably does not give the correct number of rotations between the observations, was adopted (we seek only the orientation of the spin vector, not the magnitude), and the position of the reference round depression (Thomas et al. 2007) was maintained at $0^{\circ}$ longitude. This solution involves data from four cameras on three spacecraft (DI Flyby and Impactor craft, and the SDN spacecraft). As of this writing some discrepancies remain between the body reference centers for the three missions and introduce relative errors of $\sim 100 \mathrm{~m}$ in positions of some control points determined by one camera and limbs measured in another. In the region of overlap of the image coverage near the impact site, the effect is to make projections of points from SDN and DI vary in position by up to 10 's of $\mathrm{m}$. For change comparisons we further project DI images relative to SDN images to allow better comparison of some changes which are of the $20 \mathrm{~m}$ scale.

The best effective spin pole orientation is found to be $R A=255^{\circ}$, Dec. $=64.5^{\circ}$. An uncertainty of $\sim 3^{\circ}$ on the sky is estimated in this direction due to the mission-to-mission geometric discrepancies. This spin orientation produces an obliquity of $16.4^{\circ}$. Perihelion occurs with a sub solar latitude of $4^{\circ} \mathrm{S}$.

The new estimate is $\sim 16^{\circ}$ different from the DI estimation of $\mathrm{RA}=293.8^{\circ}$, Dec. $=72.6^{\circ}$. Chesley et al. $(2012)$ have shown that the new shape and orientation predict light curves that better account for the extended photometric time series obtained just prior to DI encounter and that precession must be very small. Apparently the former results, which involved the simultaneous determination of a rotation vector and limb shape "found" the wrong solution.

\section{Shape and topography}

The shape model (Fig. 1b) is well constrained by the new image data (the total image coverage is now $\sim 2 / 3$ of the object) and by limb silhouettes from the 2005 DI data. The shape is almost pyramidal from some perspectives (Fig. 1b), is only modestly elongated, and is dominated by two gravitationally low regions, one encompassing the south pole and one at low latitudes near $270^{\circ} \mathrm{E}$. Model properties are given in Table 1 . Uncertainty in the volume is chiefly due to the small amount of unseen area and the discrepant fits of some of the data, as well as the ambiguities of limb placement in some regions. We have updated the mean density solution of Richardson et al. (2007) of $400 \mathrm{~kg} \mathrm{~m}^{-3}$ using the slightly smaller volume and its uncertainties. There is a large high end uncertainty on the mass due to uncertain effects of nucleus outgassing on particle trajectories (Richardson et al., 2007). The updated density is $470+780-230 \mathrm{~kg} \mathrm{~m}^{-3}$. With a mean density of $470 \mathrm{~kg} \mathrm{~m}^{-3}$ surface accelerations range from 0.030 to $0.038 \mathrm{~cm} \mathrm{~s}^{-2}$.

The maximum model moment of inertia direction is $\sim 9^{\circ}$ from the calculated rotation axis (Table 1); this offset is three times greater than the estimated error of the direction of the spin axis. There is no evidence for appreciable excitation of the spin state (Belton et al., 2011 ) that could lead to a separation of these two directions. We estimate the uncertainty in the orientation of the moments in a homogeneous model by adding (or subtracting) volume from the areas not well constrained by the imaging, most notably in the
Table 1

Tempel 1 properties.

Mean radius: $2.83 \pm 0.1 \mathrm{~km}$

Volume: $95.2 \mathrm{~km}^{3}$, area: $108 \mathrm{~km}^{2}$

Range of radii: $2.10-3.97 \mathrm{~km}$

Effective spin pole between observations of July 2005 and February 2011: $\mathrm{RA}=255^{\circ}$, Dec.$=64.5^{\circ}\left(15.9^{\circ}\right.$ different from previous value of $293.8^{\circ}$, $\left.72.6^{\circ}\right)$

If density is $470 \mathrm{~kg} \mathrm{~m}^{-3} \mathrm{~g}$ at the surface is $0.030-0.038 \mathrm{~cm} \mathrm{~s}^{-2}$

Inertia principal axis orientations: $\mathrm{A}:-2.7^{\circ}, 16.8^{\circ} \mathrm{E} ; \mathrm{B}:-3.4^{\circ}, 107.0^{\circ} \mathrm{E} ; \mathrm{C}$ : $+80.6^{\circ}, 247.5^{\circ} \mathrm{E}$

Principal moment ratios: A/C: $0.688 ; \mathrm{B} / \mathrm{C}: 0.930$

region $120-190^{\circ} \mathrm{E}$. The limb traces (Fig. 1) are somewhat dependent upon the model itself, and volume can be added in this area if the limb's position on the surface is moved (notably in the HRI departure images). The amount of volume that can be added in this area is restricted by the terminator views in the outbound SDN data as well as the HRI limb data. We find that addition of up to $300 \mathrm{~m}$ in a band near $20^{\circ} \mathrm{N}, 180^{\circ} \mathrm{E}$ can move the maximum moment model latitude to $85.6^{\circ}$. Thus, with a $3^{\circ}$ uncertainty in the spin vector, we could have a moment/spin discrepancy of $\sim 1-7^{\circ}$. Pending further work on resolving the inter-mission geometry misfits, we leave open the possibility of internal inhomogeneity of mass distribution, although it appears no large amount is required.

\section{Geological features}

The images, mapped in Fig. 2a, show that the surface of Tempel may be broadly divided into two terrain types: rough, pitted terrain, and relatively smoother areas that include a range of topography. Fig. 2b shows a sketch map of major surface features, and Fig. 2c shows the distribution of gravitational heights, nearly identical to heights above an equipotential. Fig. $2 \mathrm{~d}$ shows a polar projection of the image map at high southern latitudes.

\subsection{Pitted terrain}

Rough, pitted regions occupy much of the surface of the comet nucleus, and somewhat resemble the pitted terrain on comet Wild 2 (Brownlee et al., 2004). Only one of the four smooth regions has pits. There is a variety of sizes, sharpness, and completeness of pits with regions of overlapping depressions forming rough, jumbled topography. Other areas have discrete, nearly rimless depressions, and some pits are expressed primarily as topographic and albedo rings with probable fill on the inside (Fig. 3; Thomas et al., 2007). A few have distinct flat floors and some have albedo indicators of downslope motion of debris on the walls (Fig. 3b). Hummocks occur on some pit floors. Many of the pits mapped in DI data have smooth rims and somewhat annular albedo patterns (Thomas et al., 2007). While the largest pits are several hundred meters across, few are more than $250 \mathrm{~m}$ in diameter. Depths can be as much as $25 \mathrm{~m}$, most pits are well below $15 \mathrm{~m}$ in depth based on stereo and shadow measurements.

We have measured the diameters and locations of clearly discernable pits (done by PT and AQ; mapped in Fig. 2b). Because of the overlapping and partial exposure of many pits, as well as the usual problems of rapidly changing viewing conditions of data taken on flybys of irregularly-shaped bodies, our statistics are necessarily approximate. We have also mapped pits on Wild 2. We have mapped all rounded depressions as one population, including the raised-rim features near the DI impact site, realizing that one or more might be expected to be impact craters (Thomas et al., 2007; Schultz et al., 2007; Brownlee et al., 2004) and other cryovolcanic collapse features (Belton and Melosh, 2009). Belton et al. 

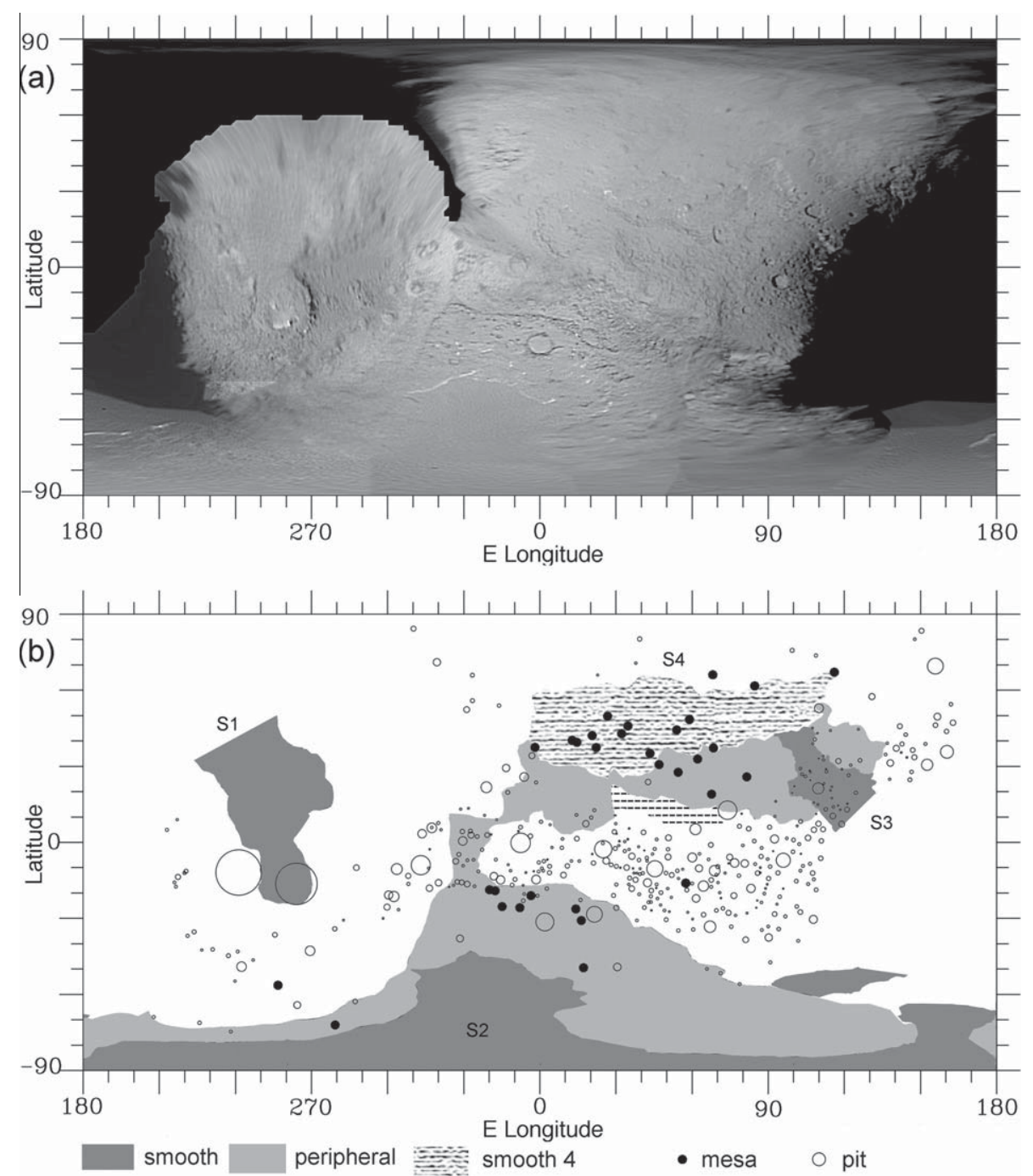

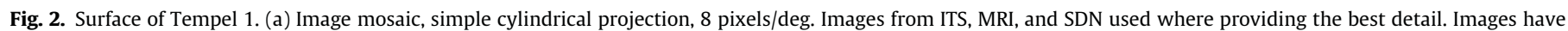

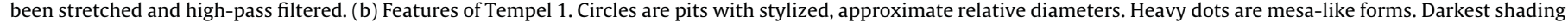

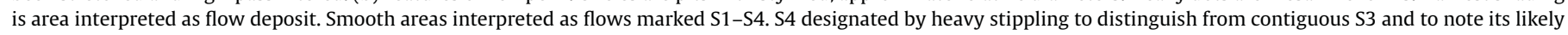

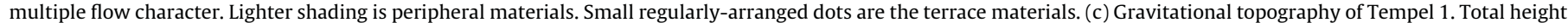


Melosh (2009) is close to the 180E mark.

(2012) ascribe $>90 \%$ of the pits to cometary outburst activity in which case a single population is appropriate. Some depressions appear to be intermediate between these raised rim forms and full depressions, suggesting a range of effective morphologies rather than separate, discrete populations. Cumulative size-frequency plots in Fig. 4 show that at well-resolved sizes Wild 2 has a more dense covering of pits. Image data for Wild 2 were dominantly $15-21 \mathrm{~m} /$ pixel and $11-40^{\circ}$ phase; for Tempel 1 they are $9-15 \mathrm{~m} /$ pixel and $20-70^{\circ}$ phase. Reliable recognition of craters or the like usually requires 5-7 pixels/diameter, so these data are good to crudely $60-80 \mathrm{~m}$ for Tempel 1 and $90-120 \mathrm{~m}$ for Wild 2 . Wild 2 data have a more gradual roll-off at smaller sizes that might extend above the expected level of resolution effects, but probably not by a significant amount. For the four largest size bins, slopes on both objects are $\sim-2.1$. Including Wild 2 data down to $200 \mathrm{~m}$ and Tempel data to $50 \mathrm{~m}$ gives cumulative $\log$ slopes of -1.7 and -2.1 , respectively. Relative to the object size the Wild 2 depressions are substantially more prominent by their size and numbers, and to some extent by the sharpness of some of their edges.

\subsection{Smooth terrain}

There are four regions of smoother terrain on Tempel 1, two observed by DI, and the third, and fourth (the largest), imaged by SDN (Fig. 5). These regions occupy topographic lows (Fig. 2b and c). Details of the slopes and relations to particular parts of the smoother regions are discussed below in Section 6. Smooth terrain lacks a dense cover of overlapping pits and has a range of surface textures from smooth at the available resolution to roughness at the $30-\mathrm{m}$ scale. We sub-divide the smooth regions into three parts: (1) those that show some suggestions of flow, (2) peripheral regions of smooth to hummocky and scabrous topography, and (3) one region of terraced materials.

\subsubsection{Probable flows}

Regions S1 and S2 (Figs. 2b and 5) were interpreted from DI images as deposits emplaced by flows on the basis of several characteristics: S1 is smooth, distinct, widens downslope, and appears laterally confined (Figs. 2 and 5). S2 shows subtle albedo markings 


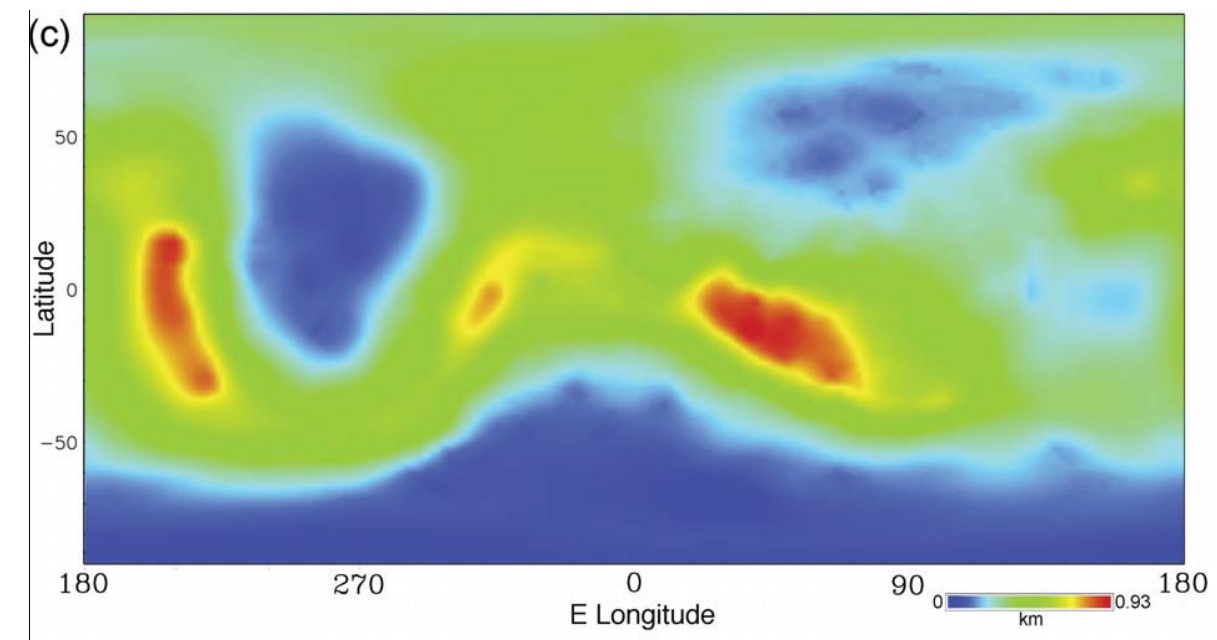

(d)

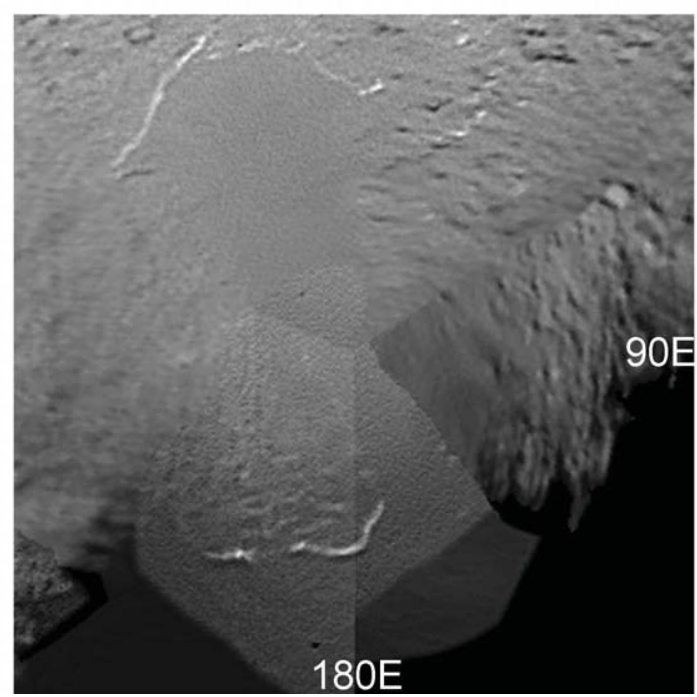

Fig. 2 (continued)

interpreted as flow toward the end defined by a scarp and adjacent hummocky terrain (Thomas et al., 2007). Most of this feature was previously interpreted to have slopes of $\sim 5^{\circ}$ away from the South polar region. Topography and slopes on the smoothest part of this feature must be interpolated from the edges as no reliable stereo control is obtained from the nearly featureless surface.

Feature S3 resembles a digitate flow; the terminator region is gravitationally higher than the western, tapering end. It differs from the other features interpreted as flows by having several pits, some flat-floored, that suggest a layer selectively being removed from or collapsing into the substrate.

Feature $\mathrm{S} 4$ is the most difficult of the smooth regions to map; as such it is mapped distinctly in Fig. 3b. The southern margin of S4 is a crenulated to digitate scarp; much of the rest of the mapped boundary is gradational and subject to interpretation (Fig. 2b and c). The surface of this region includes some mesas, and thus it is in some ways intermediate in morphology between the well-defined possible flows and the scabrous peripheral regions. Within the mapped region are subtle, anastomosing albedo patterns suggesting an east-west, or west-east fabric. This area is likely to be the remains of deposits from a multiplicity of flows.

Of the interpreted flows, only S1 (Figs. 2b and 5) displays a probable distinct source: the interior of one of the largest round depressions (including one of the few examples of exposed ice; Sunshine et al., 2006). A possible source for S2 has been suggested to be near the South pole (Belton and Melosh, 2009), but the obscured viewing and terminator position in DI images preclude positive identification. Flow S3 appears to extend over the terminator observed by SDN, perhaps near possible parts of S2, but this area between S2 and S3 remains unobserved.

Areas interpreted as flows cover about $11 \mathrm{~km}^{2}: \mathrm{S} 1: 3.7 \mathrm{~km}^{2}, \mathrm{~S} 2$ : $1.9 \mathrm{~km}^{2}, \mathrm{~S} 3: 1.1 \mathrm{~km}^{2}, \mathrm{~S} 4: 4.0 \mathrm{~km}^{2}$. If the average thickness of these is $\sim 10 \mathrm{~m}$, then individual flow volumes would be $\sim 10^{8} \mathrm{~m}^{3}$, or $10^{-1} \mathrm{~km}^{3}$.

\subsubsection{Peripheral materials}

Areas S2 and S4 have prominent adjoining regions of hummocky to scabrous topography, often with scattered mounds, some flat-topped (at least one even appears in stereo to have a small pit on top, Fig. 3d). The region adjacent to S2 includes the DI impact site, and appears to be an area of stripping of materials, possibly the remains of a previous further extent of S2 (Schultz et al., 2007; Thomas et al., 2007). South of S4 the peripheral material extends west and merges with that extending from S2 (this merging is visible at high emission angles and does not map well into the image map in Fig. 2). Some of the peripheral materials near S4 appear to have discrete different heights separated by low scarps or ridges. Notable in the area between S2 and the peripheral materials near S4 is a region of pits that are in places close-packed, and which have the lower albedo associated with some rimmed pits 

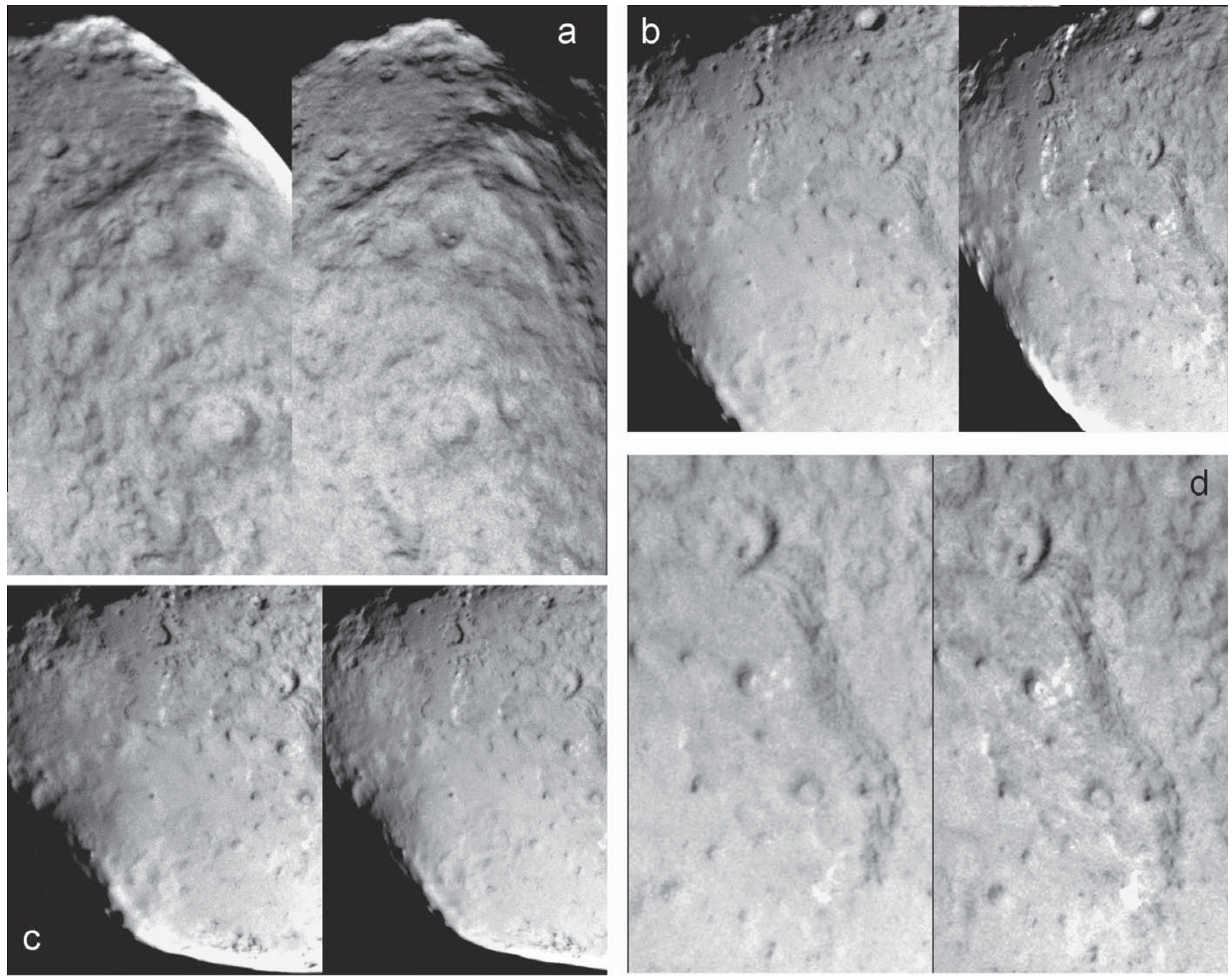

Fig. 3. Stereo views of portions of Tempel 1 . In all pairs north is roughly to the upper right. (a) Images n 30037 and $n 30038$. Center is about $20^{\circ} \mathrm{N}, 10^{\circ} \mathrm{E}$. Width of view is about

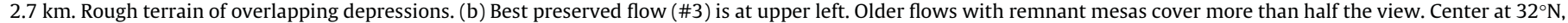

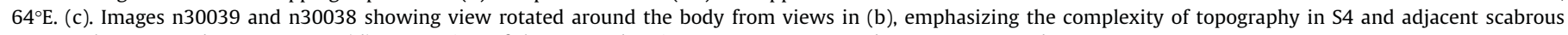
topography. Centered at $47^{\circ} \mathrm{N}, 62^{\circ} \mathrm{E}$. (d) Stereo view of the terraced region. Images n 30037 and $\mathrm{n} 30038$. Centered at $18^{\circ} \mathrm{N}, 50^{\circ} \mathrm{E}$.

(Thomas et al., 2007, Fig. 11). Peripheral materials add about $9.5 \mathrm{~km}^{2}$ to the flow areas.

\subsubsection{Terraced area}

From $\sim 10-20^{\circ} \mathrm{N}$ and $30-70^{\circ} \mathrm{E}$ between heavily pitted terrain and the peripheral materials abutting S4 is a topographic terrace (Fig. 3d), scalloped in plan form, with exposures of up to five ridges or steps along the length of this feature (for most of the length, three or fewer steps). Stereo viewing does not entirely resolve whether these are exposed layers or slumping materials; the "steps" are only 2-5 pixels wide, the "risers" only 1-2 pixels. In one area where stereo control is good, the surface rises $\sim 50 \mathrm{~m}$ radially over a group of four layers or slumps. If layers, they would be 10-15 m thick. Terraced materials cover about $1.3 \mathrm{~km}^{2}$. The total area of mapped flows, peripheral materials, and the terraces is $\sim 22 \mathrm{~km}^{2}$. There is considerable area in northern latitudes and high E longitudes (Fig. 2; bottom of Fig. 3c) seen at high emission angles that might fall into this classification with better viewing.

\subsection{Photometry of surface units}

The surface of Tempel 1, as with other comets, is dark, averaging a geometric albedo of $\sim 0.06$, with modest variations over the sur- face, and no geologic definitions possible on the basis of photometric properties (Li et al., 2009, 2012). The side observed only by SDN shows no indications of the high albedo patches seen by DI and determined to be exposures of dirty water ice (Sunshine et al., 2006). SDN did not observe the regions in which the bright patches were observed by DI.

\section{Interpretations 1: deposits and pits}

\subsection{Smooth areas: probable flows}

Initial measurements of S1 and S2 showed a slope of a few degrees in the suspected flow direction for $\mathrm{S1}$, and for much of the length of S2 (Thomas et al., 2007). The SDN data indicate a more complicated relationship of slopes and possible flows. Profiles are plotted and mapped in Fig. 6, along all four possible flows, and across them, peripheral materials, and their confining topography.

In flow $S 2$, the lowest point is at $\sim 63^{\circ} \mathrm{S}$, marked by an X in Fig. 6. Slopes south of that point along the length of the flow are $\sim 5-6^{\circ}$ (left side of profile A, Fig. 6a). There is a rise northward within the $S 2$ region, continuing through the peripheral scabrous topography above the equator to elevations considerably above the 


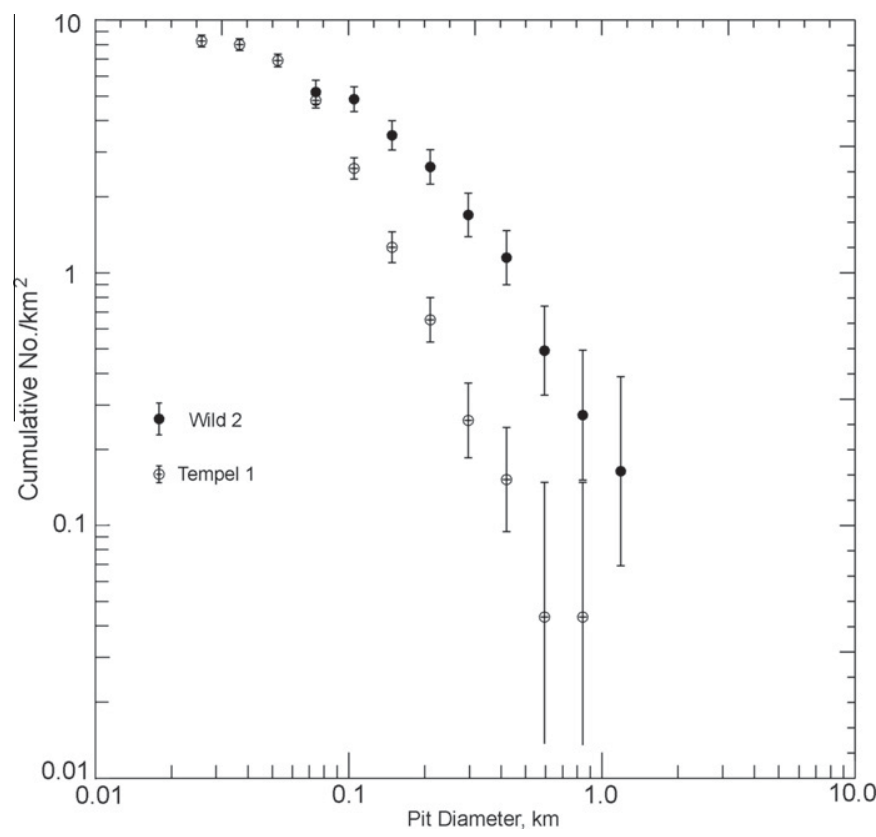

Fig. 4. Cumulative area densities of pits on Tempel 1 and Wild 2. Error bars are simply $\sqrt{ } n$-based.

smooth flow morphology. Profiles C and D in Fig. 6b, and the map in Fig. 2 show the lateral confinement of the flow and the flanking materials. Flow S2 also shows forms that suggest levees: deposits on the sides of flows that originate from reduced flow at the margins or overflow of a confined flow. These may reach $10 \mathrm{~m}$ above the surroundings (their smooth surfaces make stereo solution unreliable) and extend for $\sim 1.3 \mathrm{~km}$. There is smooth material extending laterally beyond the ridges, which might result from le- vees being buried by a subsequent, more extensive flow. The profiles indicate a slight slope $\left(\sim 2^{\circ}\right)$ crosswise to the putative flow direction. The control points are determined well enough in the surroundings that this apparent slope is real.

S3 shows a slope along its length of $\sim 4^{\circ}$. It is also well confined laterally (Fig. 6c). The higher end essentially at the terminator, shows shallowing, and this area might be close to its source.

S4 slopes in the opposite direction (west to east) from S3 at 6$7^{\circ}$ for $2 / 3$ of that distance (Fig. $6 \mathrm{~d}$ ). It is also laterally confined, but has a substantial crosswise asymmetry of about $4^{\circ}$. The minimum for the S4 profile occurs at longitude $\sim 80 \mathrm{E}$. Because this area is interpreted to be remnants of several flows actual individual lengths and slopes may be camouflaged.

The $\mathrm{S} 1$ is the simplest, and may be a single flow with a specific source. The S2's full extent and source(s) are less clear. The peripheral materials, including some poorly-imaged complicated smooth and jumbled areas between 90 and 140E (Fig. 2) account for $6.6 \mathrm{~km}^{2}$ in addition to the flow's $1.9 \mathrm{~km}^{2}$. The peripheral materials might indicate a much larger previous extent of a flow deposit, and perhaps compaction following deposition that resulted in a deposit with a bowl-shape rather than a level, pond-like surface. Belton and Melosh (2009) interpreted the circular depression near the apparent source as a collapse feature that was formed after the extrusion of the material in the flow.

S3 also appears simple, but presents the distinction of having pits. There are few enough pits that statistical comparison with other populations on Tempel 1 are not warranted. The largest pit in the flow (Fig. 3b) is irregularly shaped and has a flat floor. Shadow lengths in pits in this flow indicate depths of $4-16 \mathrm{~m}$. The margins appear crisp, but this may be erosional action on slightly inhomogeneous materials. The pits might reflect external effects such as sublimation on the flow, or effects from the flow covering pre-existing topography in the underlying materials. The pit and flow relation is discussed more in Section 6.2.

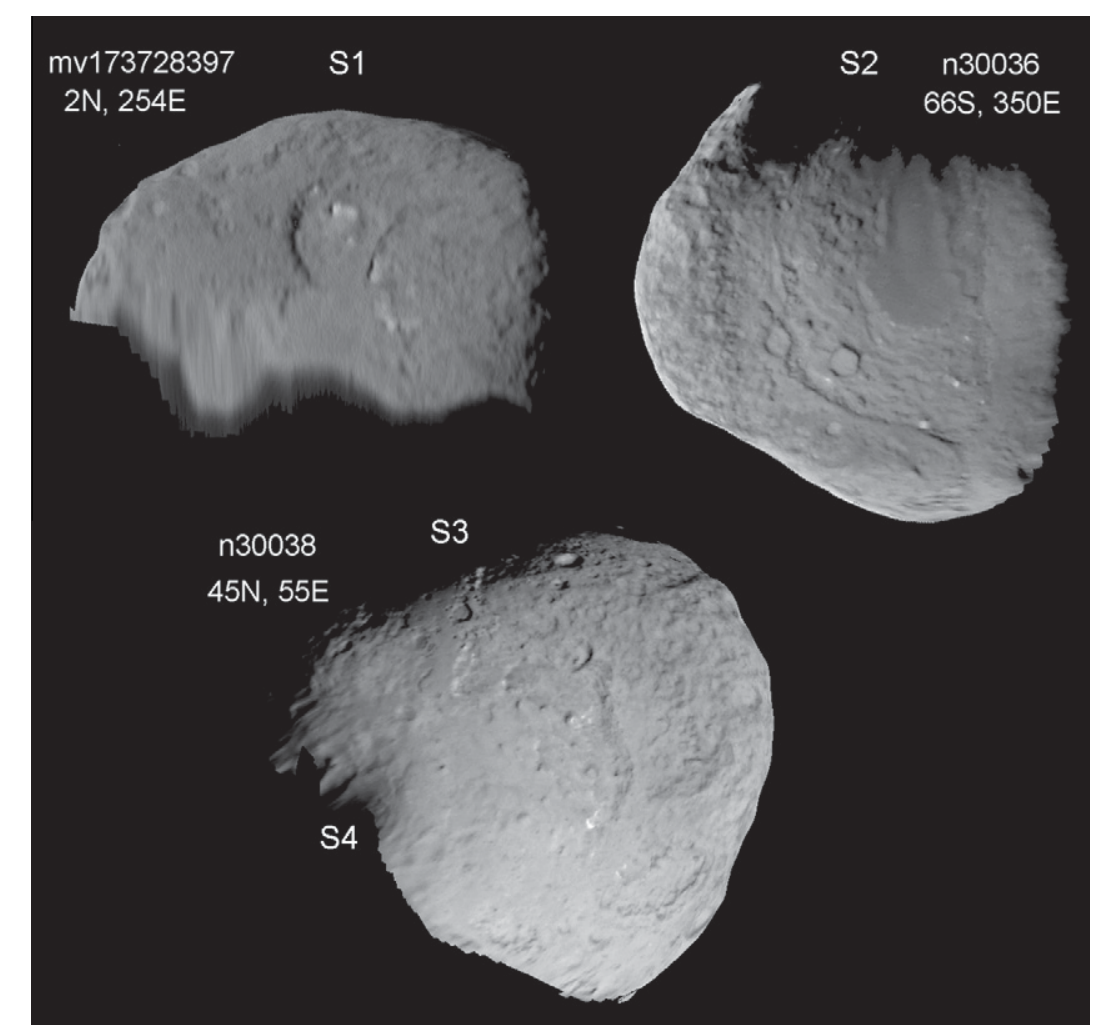

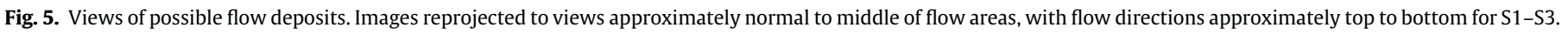



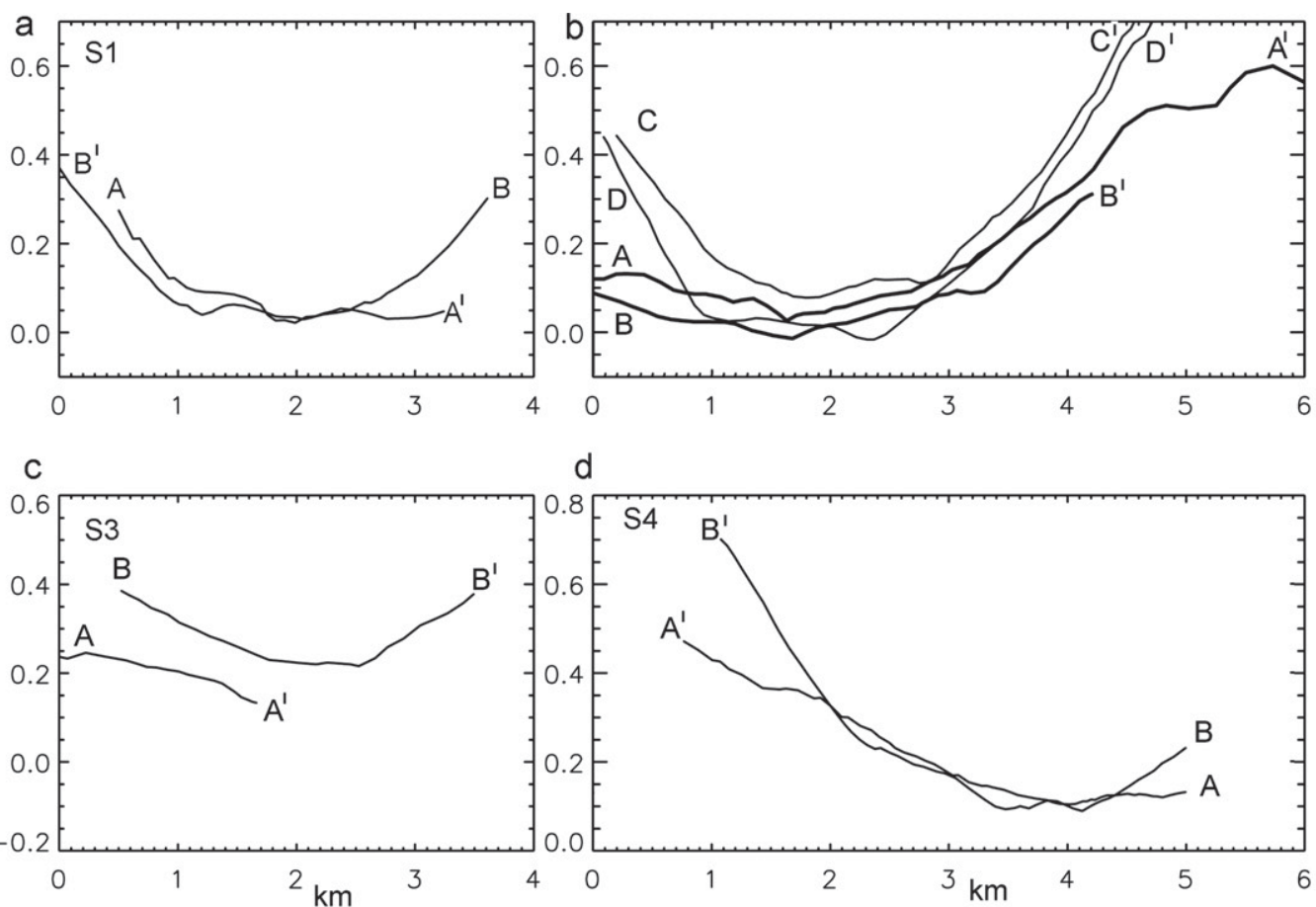

d

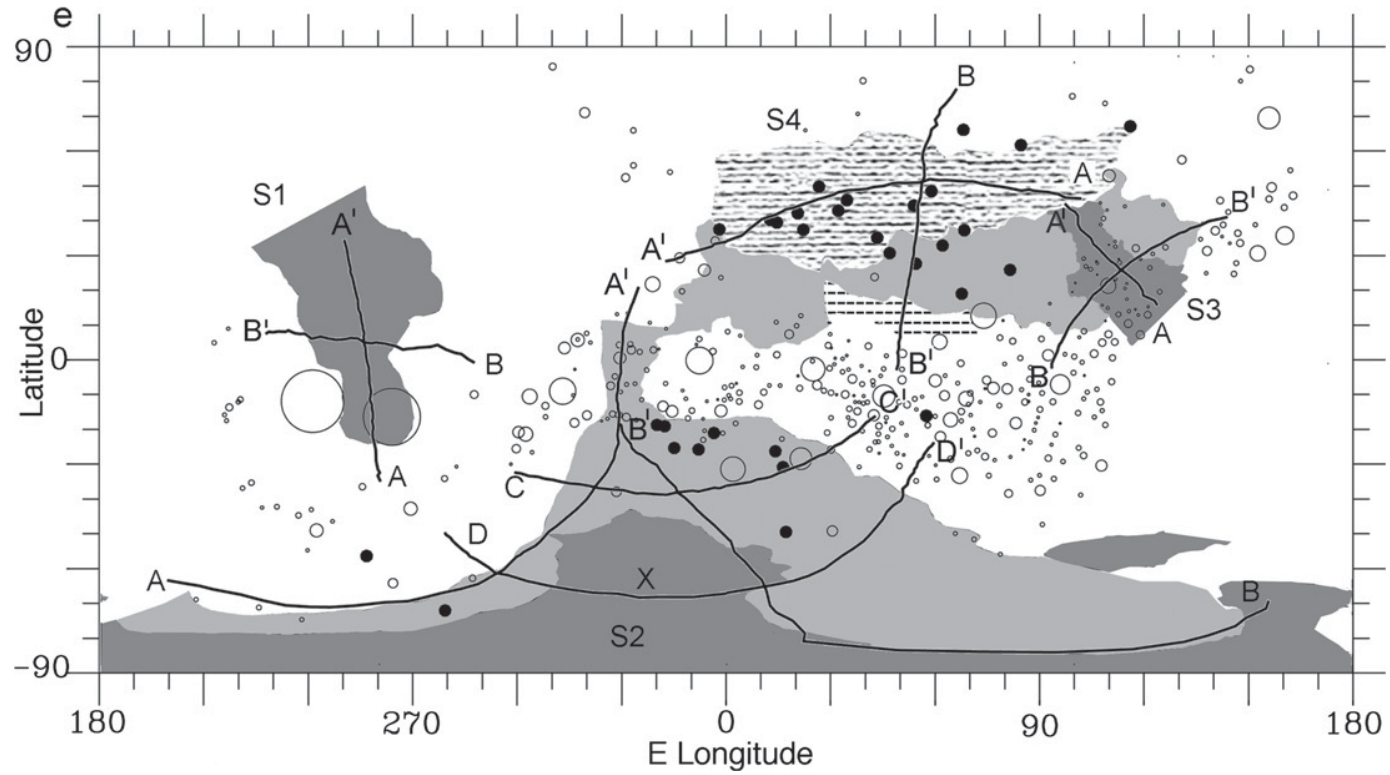

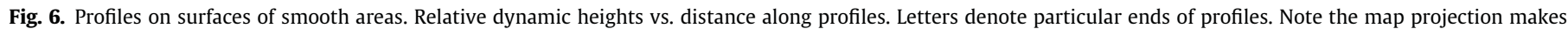

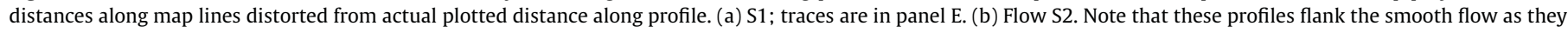

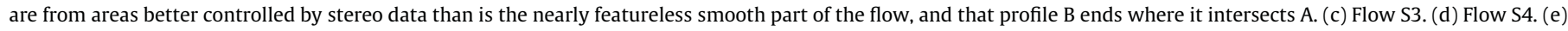
Map from Fig. 2 with profile locations.

S4 is probably several moderately eroded flows (Figs. 6 and 7), as is some of the surrounding material. The mesas are definite indicators of removal of material, and the varied roughness of S4 and the peripheral materials is a strong indicator of multiple deposits and extended removal. The tallest mesas measure about $25-30 \mathrm{~m}$ (from stereo measurements) in height. The cumulative size distribution of the mesas has approximately a -2 slope in logarithmic space. While this slope is not well constrained because of the few data, it does indicate there is no preferred horizontal dimension to the mesas. A preferred size might be expected if they were constructional forms with deposition from a central source.

Thus, while it appears there are two simple, downslope flow deposits (S1, S2), two others are intimately connected with erosion and involve much more extensive related peripheral deposits that reach up to $200 \mathrm{~m}+$ in elevation above the smoother flow sections (Fig. 6). In fact, for S2, the previous interpreted source (Belton and Melosh, 2009) is only slightly above the lowest point in a topographic bowl opening toward the south polar region The outer boundaries of the annular materials associated with S2 and S4 do not follow level lines (Figs. 6 and 7). The upper part of S2 and the surrounding materials average about $200 \mathrm{~m}$ lower than most of the perimeter of the periphery, some of which is $\sim 700 \mathrm{~m}$ above the lowest point. Thus, the mapping and modeling do not show the smooth flows and peripheral materials to be simple compacted ponds of material. Because it is bounded by an eroding scarp (Section 7) the smooth section of S2 has undoubtedly been more extensive in the past, but how far toward the north it may have extended is not clear, though the simple image interpretation indicates 




Fig. 7. Areas of smooth terrains on topographic map. Possible subdivisions of flow and peripheral materials are noted in region S4.

$1.2 \mathrm{~km}$ at most. The peripheral materials are clearly associated with the probable flows, but the wide topographic differences between the flows and the margins of the peripheral materials complicate the interpretation. The peripheral materials might derive from a high-velocity, thinner deposition facies, but because some of the peripheral material is higher than the proposed source, this explanation also is at best incomplete.

The terrace units may also indicate deposits well over $50 \mathrm{~m}$ in total thickness. We do not know the former extent of these deposits that seem to be reduced to this small "onlap" area, but from the size of other flows, material this material might have been removed from an area of more than $2 \mathrm{~km}^{2}$.

\subsection{Pits}

The discussion in Section 5.1 above has cast all round depressions including the rimmed, darker forms near the impact site, into one group, as there appears to be a full gradation of forms. The expected number of $50+\mathrm{m}$ depressions, if made by impacts by asteroidal material in a likely maximum dynamical lifetime of a Jupiter family comet of $\sim 30,000$ years is $<2 \%$ of our total (Belton

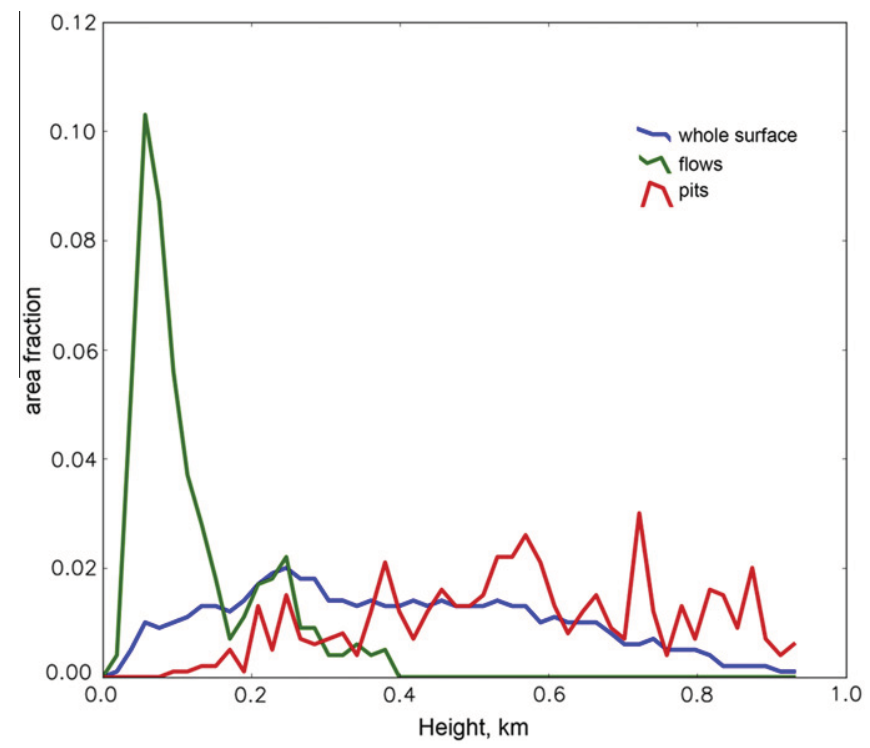

Fig. 8. Height distribution of occurrences of smooth flows, pits, and the whole surface of Tempel 1. Data are in 50 bins spanning the dynamic height range of $0.93 \mathrm{~km}$. et al., 2012). Yet the rimmed features, and some of the mesa-like forms in the area viewed by SDN, suggest processes that make resistant round forms, not just the excavation of pits. Belton and Melosh (2009) suggest cryo-volcanic collapse associated with emplacement of flows on the surface as one such process. If the majority of pits are related to outburst events and associated jet activity, then they may be subject to physical or chemical action affecting the region around the flow, possibly resulting in more resistant annular zones. Resistant tubular portions of a deposit could erode to rims, small butte-like forms, or raised mesas with included depressions (Fig. 3). Erosion of materials with such resistant zones leave very similar looking remnants on Earth, and possibly Mars (Chan et al., 2010), though obviously involving vastly different chemistry from processes on cometary nuclei. Thus, even for the rimmed remnants, we conclude there is no need to invoke processes other than those involved in endogenic pit formation: volatile loss and transport from depth, with ongoing sublimation effects at the surface.

Is the pitted terrain a general background topography being covered by the accumulating flows (and the associated peripheral materials), or are the pits also geographically or topographically limited? The geography shown in Fig. 2 suggests a global occurrence, allowing for areas not well imaged, and possibly a preference for topographically higher elevations. We have plotted the distribution of the heights of flows and of pits, compared to that of the nucleus as a whole in Fig. 8. The smooth flows are concentrated in the lowest parts as expected. The pits are somewhat more prominent at the highest areas, but their occurrence frequency does not drop significantly until the bottom third of the height range, where flows start to show significant covering. This distribution suggests that the pits are a background phenomenon being covered in places by depositional accumulations. The possible covering of pits by part of flow S3 is consistent with this scenario. Of course, once a region is covered, the change in thermal regime would over time affect processes at depth; depending on the physical differences between the covering materials and previously pitted area, pitting might continue or might be shut off.

\section{Changes between Deep Impact and Stardust-NExT}

\subsection{Observations of changes}

The Deep Impact and Stardust-NExT flybys were separated by one comet orbit, or 51/2 years. More precisely, DI imaged the comet 1 day before perihelion passage, SDN observed it 34 days after the 
next perihelion. Thus our exact time base is slightly longer than one perihelion heating period, but for simplicity we use 1 orbit for approximate calculations below. The region encompassing area S2 was seen by both DI and SDN, though at different viewing geometries. DI with three cameras obtained some coverage by the impactor camera at $1 \mathrm{~m} /$ pixel; more was covered at $\sim 10 \mathrm{~m} /$ pixel from the flyby spacecraft cameras. SDN obtained images as good as $11 \mathrm{~m} /$ pixel, but at lower phase angles than DI. Convergence angles between DI and SDN for the area near the impact site are as small as $55^{\circ}$ for a few images, but the best resolution comparison is at $72-94^{\circ}$ convergence.

Because of the change in viewing conditions between the missions, small features can be difficult to identify in both views, so there are errors in the shape model in some areas of $\sim 20 \mathrm{~m}$. The SDN image registration was refined by rubber-sheet distortion to match additional reliable common points.

The different viewing and illumination conditions at the two encounters (Fig. 9) require caution in evaluating apparent changes. There are no obvious changes between missions in albedo on scales of a hundred meters or more. A detailed photometric analysis of the overlap area by Li et al. (2012) confirms that no large-scale changes in albedo or photometric function (sensitive to surface texture) have occurred. The larger dark circular markings near the DI impact site appear unchanged. Several small brighter albedo spots $(<30 \mathrm{~m})$ in the region have disappeared, and others have appeared. A few of these may have changed in contrast and extent. None of the small brighter spots have the high albedo characteristic of the water ice exposures identified by Sunshine et al. (2006) in the DI data. Given the small size of these features and the limited resolution of the images, (at best $11 \mathrm{~m} /$ pixel) it remains uncertain that these are true surface changes rather than effects of the differences in illumination and viewing conditions between the two image sets.
However, significant changes are visible along parts of the scarp bounding the prominent flow in Fig. 9. In addition to subtle recessional changes along most of the bounding scarp, at least two crudely triangular salients evident in 2005 have disappeared by 2011 representing a surface area of $\sim 1.6 \times 10^{4} \mathrm{~m}^{2}$. In these two areas the scarp has retreated by more than $50 \mathrm{~m}$. If the subtle changes visible along most of this scarp amounted to a pixel on average, or about $10 \mathrm{~m}$, and estimating that active scarp retreat occurred along at least $1000 \mathrm{~m}$ of the boundary, another $10^{4} \mathrm{~m}^{2}$ of material could have been lost. From SDN stereo coverage and shadows visible in DI images, the thickness at the flow front is generally between 8 and $15 \mathrm{~m}$, leading to volume loss estimate of at least $2-4 \times 10^{5} \mathrm{~m}^{3}$, corresponding to $8-16 \times 10^{7} \mathrm{~kg}$ using the average density in Table 1 . Of course, because these materials appear to be processed by having been transported, erupted, and deposited, their mean density may be different from that of the whole body.

\subsection{Interpretation and context}

The observed backwasting appears concentrated in some of the brighter, better defined sections of the scarp bounding the smooth material. The best image of this scarp is an ITS view at $\sim 9 \mathrm{~m} /$ pixel (Fig. 9). The scarp is generally facing the solar direction, but some portions are oriented nearly $90^{\circ}$ to the solar azimuth but remain relatively bright. The brighter sections may be the result of the erosion removing more of the darker lag materials either by scarp collapse or small-scale sublimation pressure removing crusts. Inherently brighter areas would not by themselves be likely to erode more quickly than darker ones unless the darker lag is thick enough to shield buried volatiles from the thermal input which could be greater in lower albedo regions.

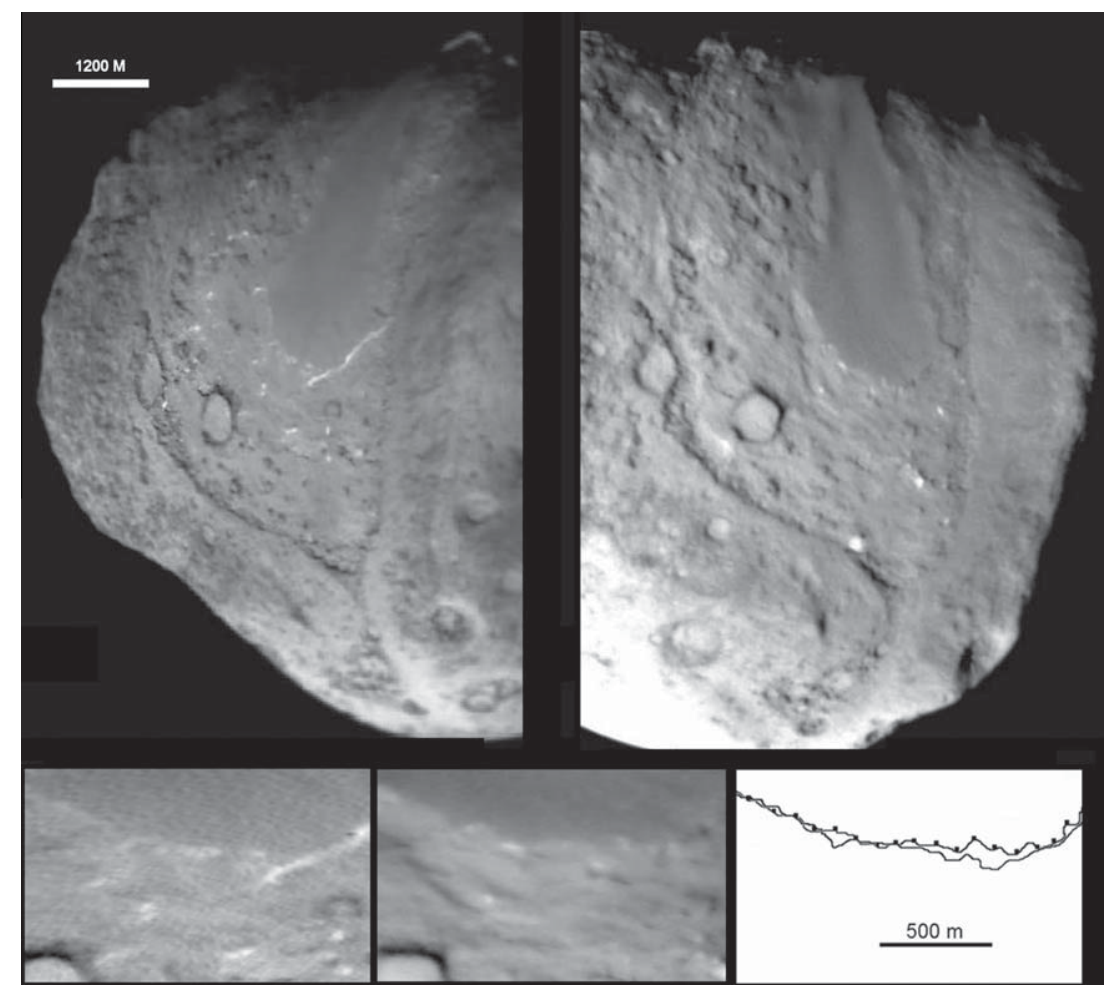

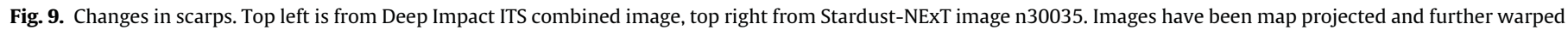

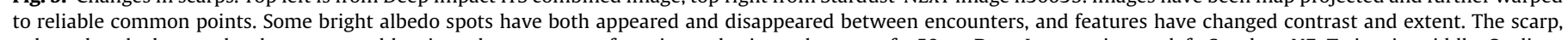

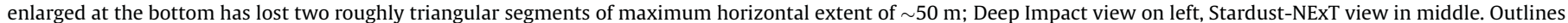

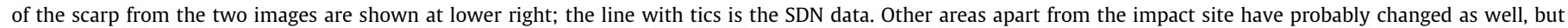
differences in lighting and viewing geometry restrict the confidence of conclusions. 
This eroding scarp is at $\sim 40^{\circ} \mathrm{S}$. With the Sun at $-4^{\circ} \mathrm{S}$ at perihelion and with an obliquity of $16.4^{\circ}$, this region receives moderate heating and is never in total darkness throughout a rotation. Farnham et al. (2012) note that no jets were detected in this region by SDN even though a jet at higher latitude $\left(\sim 60^{\circ} \mathrm{S}\right)$ was active and probably was subject to less surface insolation. Farnham et al. (2012) find most jet activity visible to SDN originated at low latitudes (15$30^{\circ} \mathrm{S}$ ) near the scarps shown in Fig. 3d. Thus, visible change and the source of some jets are correlated with scarps. The cause-and-effect relation is not clear, and the influence of solar heating in jet activity is not straightforward (see Farnham et al., 2012).

\section{Deep structure of Tempel 1}

Linear markings of albedo and texture on Tempel 1 were used to infer a deep structure of layering $50-200 \mathrm{~m}$ in thickness (Thomas et al., 2007). Deep structures of comets have been proposed to be the result of low velocity, accumulating collisions (Belton et al., 2007). Earlier interior models, e.g. primitive rubble-pile arrangements (Weissman et al., 2004)), do not predict layered structures. Three dimensional simulations of low velocity binary collisions by Asphaug (private communication) show the production of layers as do calculations by Lasue et al. (2011). The SDN data expand the image coverage to over $2 / 3$ the object and allow much better geodetic control on indications of 3-D structures deep in the comet. Even with this additional information, however, the usual problems of interpreting subtle markings come into play, and the extent of deep structures remains uncertain.

The linear markings so evident in the DI images at phase angles of $65^{\circ}$ and $12 \mathrm{~m} /$ pixel are invisible in SDN data at $12 \mathrm{~m} /$ pixel and $25^{\circ}$ phase. The DI images covering the layers generally have higher phase and lower emission angles than the SDN data. Layer markings mapped from DI images do appear to project on faint aligned topography visible in the SDN images.

There are hints in the SDN images of thick layering in the ridge separating the S2 and S4 regions. These are subtle alignments of topography that in stereo viewing remain marginally resolved. We have mapped them and projected them in Fig. 10. If these are indicators of different erosional modification due to different porosities, compositions, or particle sizes they suggest that the layering is a global and fundamental aspect of the structure of comet Tempel 1, and that thick layers in the comet body do not remain parallel over the whole interior. The deep interior remains an intriguing area of research on this and other cometary nuclei, beyond the limited scope of this report.

\section{Interpretations 2: evolution of Tempel 1}

\subsection{Timescales of feature change}

The two terrain types suggest either an on-going balance between sublimational erosion of pitted terrain and deposition of flows or a two-stage history of pit formation followed by dominance of eruption and deposition. The division between the two terrains is not perfect in that some pit features occur within the smoother materials, but as shown by Fig. 8 , the pits probably have developed everywhere there has been minimal covering by flow materials. Thus we need some estimates, however approximate, of possible time scales of events in the generation of the smooth areas compared to any processes going on in the pitted terrains.

Under the assumption that the pits are internally formed, we do not have a cratering record to provide any time scales. Belton et al. (2012) have modeled pit formations on assumptions relating to outburst detections and conclude that the population of pits could have formed in $<400$ orbits with minimal sublimation erosion. However, the present population may represent a balance between production and loss due to erosion and the achievement of a steady state. In this case $\sim 70 \%$ of the observed population may be achieved in $\sim 1.4 \tau_{1}$ where $\tau_{1}$ is a mean lifetime of pits against erosion. With $\tau_{1}=50-100$ orbits (Belton et al., 2012) much of what we see could have been established in as few as 100 orbits. Unfortunately the area of imaging covered by both missions includes few well-resolved pits that may be compared for changes. The essential absence of such changes on the modest number of pits compared does suggest formation time scales far longer than one orbit, probably at least tens of perihelion passages.

For the flows, we have their geometry and the one observation of change: a maximum of $\sim 50 \mathrm{~m}$ of scarp retreat, or $\sim 2$ $4 \times 10^{5} \mathrm{~m}^{3} /$ orbit. Possible lifetimes of flows can be estimated either by scarp retreat rates and distance eroded, or by a volume

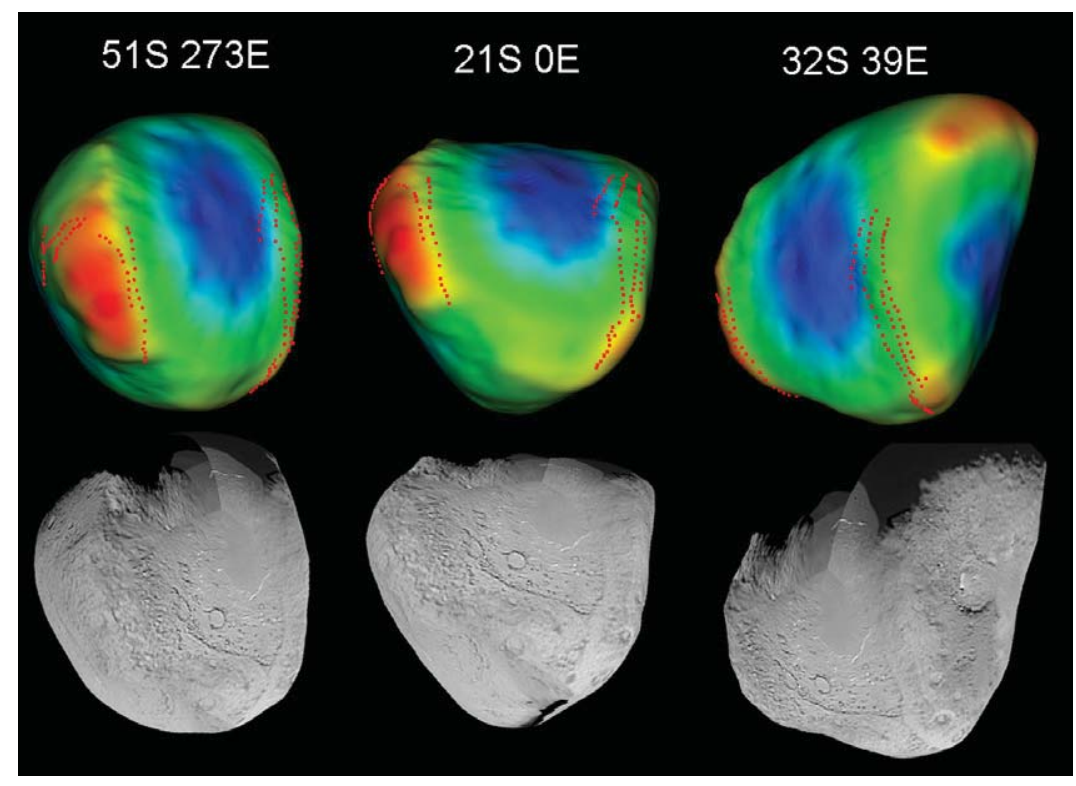

Fig. 10. Three views of mapped points along linear features. Mapped data on right side of each view are from DI; data on left side of each view are from SDN. Background colors are topography (see Fig. 2) (For interpretation of the references to color in this figure legend, the reader is referred to the web version of this article.). 
loss rate. Because we have seen the results of only one perihelion passage, and the activity of Tempel 1 has been changing (Meech et al., 2011), these estimates are crude. Treated as average scarp retreat, the observed changes are best approximated as retreat of $25 \mathrm{~m}$ /orbit. A scarp retreating a distance of $3 \mathrm{~km}$ at $25 \mathrm{~m} /$ orbit would survive $\sim 120$ orbits. This retreat rate is based on the behavior of a modest fraction of the exposed scarp, and thus an average rate might be lower, and inferred timescales longer. Additionally, the notion of a scarp uniformly retreating the entire length of the flow without edge effects is probably unrealistic. Thus the scarp retreat rate suggests a flow lifetime of 120 orbits, and perhaps much more. (We also note the observational time base is somewhat longer than one perihelion heating period, so exact calculated rates would be somewhat reduced, and calculated times for erosion thus further increased.) The volumetric estimate of lifetime would suggest that a flow of $10^{8} \mathrm{~m}^{3}$ lost at $2 \times 10^{5} \mathrm{~m}^{3}$ /orbit lasts 500 orbits. Neither of these results is likely to be a realistic estimate beyond order-of-magnitude. Furthermore, vertical loss may play a role; see Section 9.2 below.

The topography in the region peripheral to S4 suggests downward erosion of $>20 \mathrm{~m}$, perhaps several 10's of $\mathrm{m}$, over more than a $\mathrm{km}^{2}$ in an elongated region $\sim 600 \mathrm{~m}$ across. The mesas suggest that like the observed changes, much of this erosion was accomplished by scarp retreat where collapse and exposure of sublimating material could proceed. We do not know the times needed to produce the likely $5+$ layers in this area, or the erosion rate, but retreat of over $300 \mathrm{~m}$ at $25 \mathrm{~m}$ /orbit would occupy at least 12 orbits, and perhaps much more. For the region S2 periphery, the apparently eroded area is roughly $0.7 \mathrm{~km}$ in radius, and using $25 \mathrm{~m}$ /period gives 28 orbits, or at least $\sim 150$ years to reach the current state of stripping.

\subsection{Material lost in an orbit}

In order to understand the relationship of the observed changes in topography to the overall evolution of the nuclear surface, we estimate the total amount of material lost by the nucleus during an orbital period. Early estimates by Lisse (2002) and Belton et al. (2007) were based on modeling the dust at a single time and extrapolating to the entire orbit. With data now available, a far better approach is to use the observed release of water as measured via $\mathrm{OH}$ production rates by Schleicher (2007). The 2005 apparition is much better observed than any other, with 68 independent observations from 8 March 2005 through 29 September 2005 (perihelion occurred on 5 July 2005). We averaged observations on the same day to provide a set of 24 measurements and used trapezoidal integration over those points to determine the integral loss rate just in water. To extrapolate beyond those points, we assumed a linear increase to the first point beginning 80 days prior to that point and a linear decrease after the last point ending 200 days after that point. This corresponds to the observed asymmetry in the light curve about perihelion (Ferrin, 2005). The total water lost by the comet is $1.0 \times 10^{35}$ molecules, of which the extrapolations at the two ends comprise less than $15 \%$. Thus the result is not sensitive to the extrapolations unless the comet has a moderately high activity $\left(>10^{26} \mathrm{~s}^{-1}\right)$ all the way through aphelion. This result is also not very sensitive to parameters in the model, most of which are based on direct measurements. Converting this to mass loss, we find $\sim 3 \times 10^{9} \mathrm{~kg}$ of water are lost at every orbit. This mass loss just in water is four times higher than suggested by Lisse (2002).

To estimate the total mass loss, we need to add the other abundant volatiles and the dust. It is already known that in Tempel $1 \mathrm{CO}$ and $\mathrm{CO}_{2}$ are roughly $5-10 \%$ of $\mathrm{H}_{2} \mathrm{O}$ by number (Feaga et al., 2007a,b) and that $\mathrm{CH}_{3} \mathrm{OH}$ is about $1 \%$ (Mumma et al., 2005). These molecules are all substantially heavier than $\mathrm{H}_{2} \mathrm{O}$, and there are many more volatiles present at the $0.1-1.0 \%$ level by number, so we estimate that all volatiles other than $\mathrm{H}_{2} \mathrm{O}$ contribute an additional mass loss about $20 \%$ that of $\mathrm{H}_{2} \mathrm{O}$. It is much more difficult to estimate the mass loss in dust because the numbers are more sensitive to modeling assumptions, particularly in assumptions about the upper size cutoff. Thus we fall back on estimates of total dust/gas ratio. There is a wide range for these estimates and we arbitrarily assume a dust/gas ratio of $1: 1$ as a minimum and 2:1 as more likely. Thus we find a total mass loss of $7 \times 10^{9} \mathrm{~kg}$ per orbit as the minimum and $1.4 \times 10^{10} \mathrm{~kg} /$ orbit as more likely. The mass of the nucleus is $\sim 4.5 \times 10^{13} \mathrm{~kg}$ (Richardson et al., 2007), so this is a loss rate of $1.6-3.2 \times 10^{-4}$ the mass of the nucleus per orbit (not including the mass uncertainties). Using the bulk density of the nucleus from Richardson et al. (2007) and noting that, at least at the impact site, the porosity of the surface layers (75\%) is consistent with the bulk density (Ernst and Schultz, 2007), the total volume lost is $3.5 \times 10^{7} \mathrm{~m}^{3}$. For a total surface area of $108 \mathrm{~km}^{2}$, this yields a loss rate of $\sim 1 / 3 \mathrm{~m}$ per orbital period averaged over the entire surface. With the typical thickness of layers near $15 \mathrm{~m}$ this would suggest a typical lifetime for layers near 45 orbits. This estimate is similar to the estimate based on scarp retreat noted above and both processes are presumably operating simultaneously.

The above value of $7-14 \times 10^{9} \mathrm{~kg} /$ orbit may be compared to the amount estimated to be lost by scarp retreat on part of flow S2: $8-16 \times 10^{7} \mathrm{~kg}$, or a fractional range of $0.6-2.3 \%$. As this does not include a considerable additional length of scarps on the side of S3 and S4, there is the possibility that the scarp retreat could contribute up to several percent of the total mass loss.

\subsection{Some aspects of the comet's history}

The thick layers may indicate surviving effects of the cometary assembly process (Belton et al., 2007). Conversely, they might indicative of processes in a much larger precursor body. Either way, they would require some considerable erosion of the comet before reaching its present form.

The pitted terrain may largely represent one nominal style of loss of material from the nucleus, e.g., outburst activity. The inferred current global average mass loss rate of $1 / 3 \mathrm{~m} /$ orbit presumably is distributed among several local processes such as scarp retreat (Section 7), pit formation, and widespread sublimation. If anything approaching $1 / 3 \mathrm{~m} /$ orbit downwasting occurs, then modification of pits by expected local differences, might cause expansion and/or softening of the pit terrain. Pit relief of $\sim 20 \mathrm{~m}$ would probably require $\sim 60$ orbits, or 300 years for substantial changes. However, much previous time may have been spent much farther from the Sun (Yeomans et al., 2005) where erosion rates probably were far lower. Lifetimes expected for Jupiter family comets have been modeled as largely between 3000 and 25,000 years (Duncan et al., 2004). Thus, even with substantially reduced sublimation rates there may well have been time for surface evolution considerably in excess of the current topography of flows and pits.

The formation of the several flows and peripheral materials, apparently younger than much of the shaping of the comet, could conceivably have occupied periods as short as hours (Belton and Melosh, 2009), but because there are suggestions these forms include considerable erosion, the time scale of the mix of deposition and erosion may be many tens of orbits or longer. If the lifetime of a flow deposit is controlled by an erosion rate of $1 / 3 \mathrm{~m} /$ orbit, the lifetime of a typical flow would be $\sim 50$ orbits given the thicknesses inferred. As noted above, volume estimates of flow erosion give lifetimes of up to $>120$ orbits. Thus with lifetimes of $\sim 50$ orbits, and four visible flows, a simple recurrence period would be 12 orbits. There is some unseen territory on Tempel 1, but it is unlikely to harbor flows as it is relatively high terrain. A complete model of the recurrence interval from four visible flows would account for their erosional states and mutual partial coverings. 
If the smooth area deposits resulted from a change in thermal conditions, the orbital change 400 years ago (1609) of the semi-major axis from 3.5 AU to 1.5 AU (Yeomans et al., 2005) might be suspected of altering surface modification rates and styles. Whether the actual increase in heat input at the reduced semi-major axis is enough, and could have penetrated far enough in well under 400 years (>depth of pits, or $>30 \mathrm{~m}$ ) to make a fundamental change in processes, is not investigated here.

\section{Comparison of Tempel 1's surface to other comets}

A limited comparison of Tempel 1's pits with those of Wild 2 was made in Section 5 above. Additionally, in the visible half of Wild 2 there are no features similar to the flow-like areas on Tempel 1. Some large depressions on Wild 2 have flat floors that might be local accumulations of debris, but there is not the regional division of terrains that is obvious on Tempel 1. Comparisons with Borrelly (Soderblom et al., 2004) and Halley (Keller et al., 1988) are more difficult because of much coarser spatial resolution for images of these two bodies. However, Borrelly does display mesa forms (Britt et al., 2004); Fig. 11. Jet activity on Borrelly was concentrated in the area of these mesas. These forms have been hypothesized to supply a noticeable fraction of the comet's erosion, and scarp retreat rates were estimated to be several $\mathrm{m} /$ perihelion passage (Britt et al., 2004). Borrelly shows a suggestion of a flow with annular distinct topography (Fig. 11), but these forms are only marginally resolved and in stereo pairs (Britt et al., 2004, Fig. 3) suggest a layered structure far thicker (80-100 m; Kirk et al., 2004 ) than the smooth deposits on Tempel 1. At the available resolution it does not appear that Borrelly has a two-terrain surface similar to Tempel 1. Rather it appears to have erosion of a fractured upper surface possibly involving retreat of scarps capped by lag deposits (Britt et al., 2004).

Even more distinct from Tempel 1 is 109P/Hartley 2 (A'Hearn et al., 2011). This bi-lobed object, much smaller than Tempel 1 $(0.58 \mathrm{~km}$ vs. $2.8 \mathrm{~km}$ mean radius) has rougher and smoother terrains, but they are divided by positions on the lobes, and the rougher areas are due to small mounds rather than superposed arrays of pits. Hartley 2 is relatively much more active than Tempel 1 yet does not have the active scarp erosion visible on Tempel 1.

As different as are these comet nuclei in topography, their photometric properties are similar (Li et al., 2012). Wild 2, Tempel 1 , and Borrelly all have low visible albedo of $\sim 6 \%$, with modest variations across the surface and few areas enhanced by ice exposures.

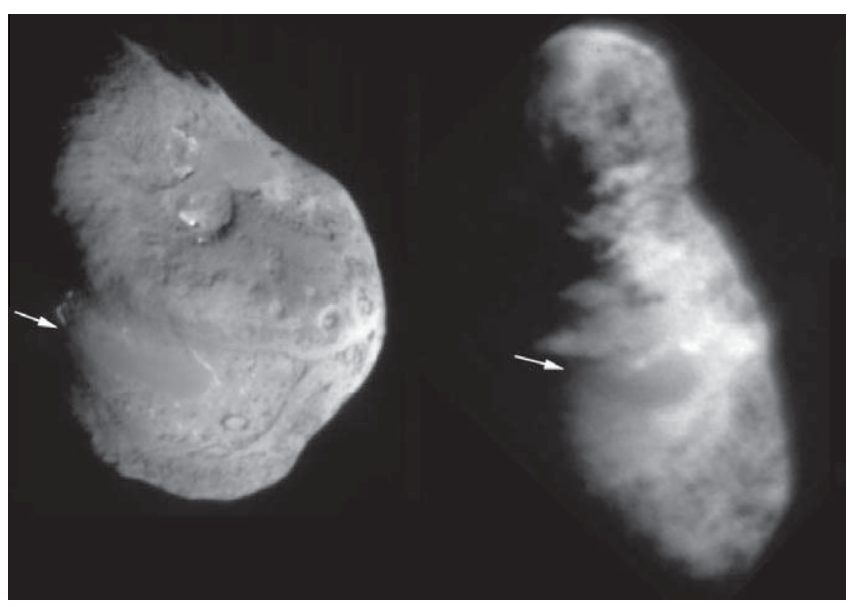

Fig. 11. Comparison of Tempel 1 and 19P/Borrelly. Borrelly image originally at $47 \mathrm{~m} /$ pixel. Arrows show smooth area S2 on Tempel 1, and a visually similar, if poorly resolved, mesa-like form on Borrelly.
The likely common near-surface processes of sublimation and lag formation that affect surface photometry clearly do not exert full control on the topographic forms on these objects.

\section{Summary}

The surface of Tempel 1 can be divided into two primary terrain types: pitted rough regions, and smoother terrain with a variety of depositional and erosional features. The pitted terrain is crudely similar to the surface of comet Wild 2, although less densely covered by pits. The smoother terrain occurs in gravitationally low regions and includes what are most probably multiple deposits from flows, manifested in 10-30 m thick units.

Erosion has proceeded in part by scarp retreat as indicated by direct comparison of images from the two flybys and by the presence of residual mesas. This backwasting may contribute a few percent of the current total mass loss from the comet. The total mass loss per orbit is estimated at $1-3 \times 10^{-4}$ the mass of the comet. This rate is fully consistent with estimated rates of landform erosion and possible lifetimes of the flows, and suggests that the apparent present combination of outbursts, erosion, and flow deposition has been operating for at least many tens of orbits and possibly hundreds of orbits.

The two-terrain character of Tempel 1 might reflect changed processes, topographically limited processes, or an interplay of erosion and deposition. The height distribution of pits suggests they are a global phenomenon, and as such may be missing in low areas chiefly because of burial.

\section{Acknowledgments}

Stardust-NExT was supported by NASA through its Discovery Program. The Science Team expresses its thanks and acknowledges its debt to the Project Management and Navigation Teams at the Jet Propulsion Laboratory, to the Deep Space Network (DSN), and to the Spacecraft Team at Lockheed Martin Aerospace (LMA) in Denver. We record our special thanks to the world-wide network of observers for providing crucial observations of Tempel 1 to support the determination of the appropriate time-of-arrival at the comet. Part of the research described was carried out at JPL under contract with NASA. Part of this research was carried out at the Jet Propulsion Laboratory, California Institute of Technology, under a contract with the National Aeronautics and Space Administration. O. Groussin's participation in the project was supported by the Centre Nationale d'Etudes Spatiales (CNES). Technical assistance was provided by K. Consroe and T. Shannon. Reviews by L. Soderblom and an anonymous reviewer notably improved the presentation.

\section{References}

A'Hearn, M.F., Belton, M.J.S., Delamere, A., Blume, W.H., 2005a. Deep Impact: A large-scale active experiment on a cometary nucleus. Space Sci. Rev. 117, 1-21. A'Hearn, M.F. et al., 2005b. Deep Impact: Excavating comet Tempel 1. Science 310 258-264.

A'Hearn, M.F. et al., 2011. EPOXI at Comet Hartley 2. Science 332, 1396-1400.

Belton, M.J.S., Melosh, J., 2009. Fluidization and multiphase transport of particulate cometary material as an explanation of the smooth terrains and repetitive outbursts on 9P/Tempel 1. Icarus 200, 280-291.

Belton, M.J.S. et al., 2007. The internal structure of Jupiter family cometary nuclei from Deep Impact observations: The talps or "layered pile" model. Icarus 187 332-344.

Belton, M.J.S. et al., 2011. Stardust-NExT, Deep Impact, and the accelerating spin of 9P/Tempel 1. Icarus 213, 345-368.

Belton, M.J.S. et al., 2012. Geologic signature of outbursts in Stardust-NExT images and a mechanism for outbursts on 9P/Tempel 1 and $17 \mathrm{P} /$ Holmes. Icarus, submitted for publication.

Britt, D.T., Boice, D.C., Buratti, B.J., Campins, H., Nelson, R.M., Oberst, J., Sandel, B.R Stern, S.A., Soderblom, L.A., Thomas, N., 2004. The morphology and surface processes of Comet 19/P Borrelly. Icarus 167, 45-53. 
Brownlee, D.E. et al., 2004. The surface of young Jupiter family comet Wild 2: View from the Stardust spacecraft. Science 304, 1764-1769.

Chan, M.A. et al., 2010. Geomorphic knobs of Candor Chasma, Mars: New Mars Reconnaissance Orbiter data and comparisons to terrestrial analogs. Icarus 205, 138-153.

Chesley, S. et al., 2012. Rotation of temple 1. Icarus, submitted for publication.

Duncan, M., Levinson, H., Dones, L., 2004. Dynamical evolution of ecliptic comets. In: Festou, M.C., Keller, H.U., Weaver, H.A. (Eds.), Comets II. University of Arizona Press, Tucson, pp. 193-204.

Ernst, C.M., Schultz, P.H., 2007. Evolution of the Deep Impact flash: Implications for the nucleus surface based on laboratory experiments. Icarus 190, 334-344. http://dx.doi.org/10.1016/j.icarus.2007.03.030.

Farnham, T.L., Bodewits, D., Li, J-Y., Veverka, J., Thomas, P., Belton, MJ.S., 2012 Connections between the jet activity and surface features on Comet 9P/Tempel 1. Icarus, submitted for publication.

Feaga, L.M., A'Hearn, M.F., Sunshine, J.M., Groussin, O., Farnham, T.L., 2007a Asymmetries in the distribution of $\mathrm{H}_{2} \mathrm{O}$ and $\mathrm{CO}_{2}$ in the inner coma of Comet 9P/ Tempel 1 as observed by Deep Impact. Icarus 190, 345-356. http://dx.doi.org/ 10.1016/j.icarus.2007.04.009.

Feaga, L., Groussin, O., Sunshine, J., A'Hearn, M., 2007b. Comparison of 9P/Tempel 1's spectra from Deep Impact with synthetic sodels. DPS 2007b. Orlando, October 8.

Ferrin, I., 2005. Secular light curve of Comet 28P/Neujmin 1 and of spacecraft target Comets 1P/Halley, 9P/Tempel 1, 19P/Borrelly, 21P/Giacobinni-Zinner, 26P Grigg-Skjellerup, 67P/Churyumov-Gerasimenko, and 81P/Wild 2. Icarus 178 , 493-516. http://dx.doi.org/10.1016/j.icarus.2005.05.007.

Hampton, D. et al., 2005. An overview of the instrument suite for the Deep Impact mission. Space Sci. Rev. 17, 43-93.

Keller, H.U., Kramm, R., Thomas, N., 1988. Surface features on the nucleus of Comet Halley. Nature 331, 227-230.

Kirk, R.L., Howington-Kraus, E., Soderblom, L.A., Giese, B., Oberst, J., 2004. Comparison of USGS and DLR topographic models of Comet Borrelly and photometric applications. Icarus 167, 54-69.

Klaasen, K.P. et al., 2008. Invited article: Deep Impact instrument calibration. Rev. Sci. Instrum. 79, 091301, 79pp.

Klaasen, et al., 2012 EPOXI instrument calibration. Icarus, in press.

Lasue, J., Botet, R., Levasseur-Regourd, A.C., Hadamcik, E., Kofman, W., 2011. Appearance of layered structures in numerical simulations of polydisperse bodies accretion: Application to cometary nuclei. Icarus 213, 369-381.

Li, J.-Y. et al., 2009. Photometric analysis of the nucleus of Comet 81P/Wild 2 from Stardust images. Icarus 204, 209-226.

Li, J.-Y. et al., 2012. Photometry of the nucleus of Comet 9P/Tempel 1 from the Stardust-NExT flyby and the implications. Icarus 222, 467-476.
Lindler, D., A'Hearn, M.F., Besse, S., Klaasen, K., 2012. Interpretation of results of deconvovled images from the Deep Impact spacecraft High Resolution Instrument. Icarus, submitted for publication.

Lisse, C., 2002. On the role of dust mass loss in the evolution of comets and dusty disk systems. Earth, Moon, Planets 90 (1-4), 497-506.

Meech, K.J. et al., 2011. Deep Impact, Stardust-NExT and the behavior of Comet 9P/ Tempel 1 from 1997-2010. Icarus 213, 323-344.

Mumma, M.J. et al., 2005. Parent volatiles in Comet 9P/Tempel 1: Before and after impact. Science 310 (5746), 270-274. http://dx.doi.org/10.1126/science. 1119337.

Newburn, R.L. et al., 2003. Stardust Imaging Camera, JGR 108. p. 8116.

Richardson, J.E., Melosh, H.J., Lisse, C.M., Carcich, B., 2007. A ballistics analysis of the Deep Impact ejecta plume: Determining comet Tempel 1's gravity, mass, and density. Icarus 190, 357-390.

Schleicher, D., 2007. Deep Impact's target Comet 9P/Tempel 1 at multiple apparitions: Seasonal and secular variations in gas and dust production. Icarus 190, 406-422. http://dx.doi.org/10.1016/j.icarus.2007.04.013.

Schultz, P.H. et al., 2007. The Deep Impact oblique impact cratering experiment. Icarus 190, 295-333.

Semenov, B.V., Acton, C.H., 2006. Deep Impact SPICE Kernels. V1.0, DI-C-SPICE-6V1.0. NASA Planetary Data System.

Semenov, B.V., Elson, L.S., Acton, C.H., 2004. Stardust SPICE Kernels. V1.0, SDU-CSPICE-6-V1.0. NASA Planetary Data System.

Soderblom, L.A. et al., 2004. Imaging Borrelly. Icarus 167, 4-15.

Sunshine, J. et al., 2006. Exposed water ice deposits on the surface of comet Tempel 1. Science 311, 1453-1455.

Thomas, P., 1993. Gravity, tides, and topography on small satellites and asteroids. Icarus $105,326-344$.

Thomas, P.C. et al., 2002. Eros: Shape, Topography, and Slope processes. Icarus 155, 18-37.

Thomas, P.C. et al., 2007. The shape, topography, and geology of Tempel 1 from Deep Impact observations. Icarus $187,4-15$.

Vanicek, P., Krakiwsky, E.J., 1986. Geodesy: The Concepts, second ed. Elsevier, New York, 697pp.

Veverka, J. et al., 2012. Overview of the Stardust-NExT encounter of comet Tempel 1. Icarus, submitted for publication.

Weissman, P.R., Asphaug, E., Lowry, S.C., 2004. Structure and density of comet nuclei. In: Festou, M.C., Kel ler, H.U., Weaver, H.A. (Eds.), Comets II. University of Arizona Press, Tucson, pp. 337-358.

Yeomans, D.K., Giorgini, J.D., Chesley, S.R., 2005. The history and dynamics of Comet 9P/Tempel 1. Space Sci. Rev. 17, 123-135 